\title{
Envelhecimento e qualidade de vida: análise da produção científica da SciELO
}

\author{
Aging and quality of life: Analysis of \\ scientific production in SciELO
}

\author{
Nathaly Wehbe DAWALIBI ${ }^{1}$ \\ Geovana Mellisa Castrezana ANACLETO' \\ Carla WITTER \\ Rita Maria Monteiro GOULART \\ Rita de Cássia de AQUINO'
}

\begin{abstract}
Resumo
A produção científica é um processo contínuo de descoberta e democratização do conhecimento para a comunidade científica e a sociedade. As pesquisas metacientíficas tornaram-se um instrumento importante de avaliação. Analisaram-se 69 artigos sobre envelhecimento e qualidade de vida, quanto às variáveis: título, autoria, sexo, áreas de conhecimento e periódicos. Observaram-se algumas diferenças estatisticamente significantes nos resultados obtidos: títulos com até 12 vocábulos, autoria múltipla e sexo feminino. Houve concentração da publicação nas áreas de Psicologia e de Medicina. A Revista de Saúde Pública teve predomínio na área de Ciências da Saúde em relação às Ciências Humanas. Houve adequação dos títulos e da autoria múltipla, porém, maior produção feminina. Psicologia, Medicina e a Revista de Saúde Pública foram as áreas de conhecimento e o periódico que mais publicaram sobre o tema. Concluiu-se que a avaliação sistemática da produção, desde a autoria até o delineamento, pode contribuir para estabelecer uma política de pesquisa na área.
\end{abstract}

Unitermos: Pesquisa cientifica; Qualidade de vida; Velhice.

\begin{abstract}
Scientific production is a continuous process of discovery and democratization of knowledge for the scientific community and society. Metascientific studies have become an important evaluation tool. A total of 69 articles on aging and quality of life were analyzed, considering: title, authorship, author's gender, knowledge area, and journal. Some statistically significant differences in the results were: titles up to 12 words; multiple authors and female authors. There was a concentration of publishing in the areas of Psychology and Medicine. The Journal of Public Health was predominant in the area of Health Sciences in relation to the Humanities. Titles and multiple authors were adequate, however, female production was higher. Psychology, Medicine and the Journal of Public Health were the areas of knowledge and the journal that published more on the subject. It was concluded that systematic evaluation of the production, from author to the study design, can help to establish a research policy in the area.
\end{abstract}

Uniterms: Scientific research; Quality of life; Old age.

$\checkmark \nabla \nabla$

1 Universidade São Judas Tadeu, Programa de Pós-Graduação Stricto-Sensu em Ciências do Envelhecimento. R. Taquari, 546, Mooca, 03166-000, São Paulo, SP, Brasil. Correspondência para/Correspondence to: C.WITTER.E-mail: <prof.carlawitter@usjt.br>. 
Estimativas bem estabelecidas projetam que o número de idosos até 2025 será superior a 30 milhões, e a velhice tanto poderá ser acompanhada por altos níveis de doenças crônicas quanto por saúde e bem-estar (Debert, 1999; Lima, 2003; Lima, Silva \& Galhardoni, 2008). De acordo com Neri e Guariento (2011), é importante melhorar as condições socioeconômicas, principalmente nos países emergentes, como o Brasil, para possibilitar uma boa qualidade de vida aos idosos em sua velhice.

No Brasil, assim como em diversos países em desenvolvimento, o aumento da população idosa vem ocorrendo de forma muito rápida e progressiva, sem a correspondente modificação nas condições de vida (Cervato, Derntl, Latorre \& Marucci, 2005). O aumento da população idosa brasileira será de 15 vezes, aproximadamente, entre 1950 e 2025, enquanto o da população como um todo será de não mais que cinco vezes no mesmo período. Tal aumento colocará o Brasil, em 2025, como a sexta população de idosos do mundo, em números absolutos (Kalache, Veras \& Ramos, 1987). Nas últimas décadas, esse fato tem aumentado a consciência de que está em curso um processo de envelhecimento (Neri, 2007a).

O envelhecimento pode ser definido como um processo sociovital multifacetado ao longo de todo o curso da vida. A velhice denota o estado de "ser velho", condição que resulta do processo de envelhecimento que gerações vivenciaram e vivenciam dentro de contextos sociais, políticos e individuais diversos (Lima et al., 2008; Neri, 2006)

Baltes e Smith (2006) ressaltam haver evidências de que a grande maioria dos idosos apresenta nível elevado de comprometimento funcional, dependência e solidão. Entretanto, envelhecer não é sinônimo de doença, inatividade e contração geral no desenvolvimento. Na literatura gerontológica, envelhecer é considerado um evento progressivo e multifatorial, e a velhice é uma experiência potencialmente bem-sucedida, porém, heterogênea, evivenciada com maior ou menor qualidade de vida (Lima et al., 2008; Neri, 2003; Neri, 2007b; Neri, Yassuda \& Cachioni, 2004).

Desde a década de 1980, há diversas iniciativas internacionais que valorizam a possibilidade de se considerar o envelhecimento como um processo

394 positivo, pensado como um momento da vida de bem- -estar e prazer. A política de desenvolvimento ativo, proposta pela Organização Mundial da Saúde (OMS, 2005), é um exemplo real dessas recomendações, enfatizando que envelhecer bem não é apenas responsabilidade do indivíduo e, sim, um processo que deve ser respaldado por políticas públicas e por iniciativas sociais e de saúde ao longo do curso da vida. A princípio, a criação dessa política parte do pressuposto de que, para se envelhecer de forma saudável, é fundamental aumentar as oportunidades para que os indivíduos possam optar por um estilo de vida mais adequado, que inclui mudanças de hábitos alimentares e atividade física regular e, consequentemente, o controle da saúde física e psicológica. Assim, a definição de envelhecimento ativo é apresentada como a "otimização das oportunidades de saúde, participação e segurança, com o objetivo de melhorar a qualidade de vida à medida que as pessoas ficam mais velhas" (OMS, 2005, p.13).

O conceito de qualidade de vida relaciona-se à autoestima e ao bem-estar pessoal e abrange uma grande gama de aspectos, tais como: capacidade funcional, nível socioeconômico, estado emocional, interação social, atividade intelectual, autocuidado, suporte familiar, estado de saúde, valores culturais, éticos e religiosidade, estilo de vida, satisfação com o emprego e/ou com as atividades da vida diária e com o ambiente em que se vive (Vecchia, Ruiz, Bocchi \& Corrente, 2005). Assim, o conceito de qualidade de vida é subjetivo e dependente do nível sociocultural, da idade e das aspirações pessoais de cada indivíduo (Neri, 2007b, 2007c; Vecchia et al., 2005).

Borglin, Edberg e Hallberg (2005) demonstraram que a qualidade de vida de idosos depende da preservação do self e da manutenção de objetivos ao longo da vida, e a experiência de qualidade de vida envolveria valores pessoais, experiências anteriores, capacidade de adaptação às mudanças, independência, autonomia, atividades, saúde, relações sociais e viver em casa. Neri (2007c) apresenta um modelo psicológico de bem-estar subjetivo na velhice, no qual: a relação entre os riscos associados ao envelhecimento e o bem estar subjetivo é mediada pelos mecanismos de auto-regulação do self e pelo senso de ajustamento psicológico, em interação com os recursos sociais de que o idoso dispõe (p.14). 
Nesse modelo, a autora afirma que a qualidade de vida está relacionada à "satisfação global e referenciada a domínios", que são percebidos pelos idosos de acordo com os seus afetos positivos e negativos, vivenciados ao longo da vida e no processo de envelhecimento. Esses são formados pelas interligações mútuas entre as variáveis antecedentes (riscos socioeconômicos e biológicos, tais como: pobreza, exclusão social, baixa escolaridade e baixo status ocupacional; doenças somáticas, déficits sensoriais, depressão, dor crônica, incapacidade funcional e intelectual, inatividade e susceptibilidade ao estresse crônico), moderadoras (mecanismos de autorregulação do self, tais como, autoconceito, autoestima, autoavaliação, senso de autoeficácia, senso de controle, sistema de metas, estratégias de enfrentamento, estratégias de seleção, otimização e compensação e senso de ajustamento psicológico) e de critério (o bem-estar subjetivo, que inclui a satisfação global com a vida, a satisfação referenciada a domínios e afetos positivos e negativos) (Neri, 2007c).

O World Health Organization Quality of Life (WHOQOL, Grupo de Qualidade de Vida da Organização Mundial da Saúde) $($ WHO,1998) definiu qualidade de vida como a percepção do indivíduo de sua posição na vida, no contexto da cultura e do sistema de valores nos quais ele vive, em relação aos seus objetivos, expectativas, padrões e preocupações. No processo de envelhecimento, são analisados e avaliados os seis domínios propostos pelo grupo WHOQOL, a saber: físico, psicológico, nível de independência, relações sociais, meio-ambiente e espiritualidade (religião e crenças pessoais).

Portanto, a definição de qualidade de vida é difícil, por se tratar de um constructo subjetivo e determinado por inúmeras variáveis interligadas ao longo da vida (life-span), inclusive no processo de envelhecimento humano. Compreender o envelhecimento como um processo sociovital multifacetado e se conscientizar de que se trata de um fenômeno irreversível é de suma importância para que todos, profissionais da saúde, governo, sociedade em geral e os próprios idosos, vejam a velhice não como finitude, mas como um momento do ciclo da vida que requer cuidados específicos, o qual pode e deve ser desfrutado com qualidade (Witter \& Buriti, 2011).
Dessa forma, é importante levantar e analisar a produção científica sobre envelhecimento e qualidade de vida, com o objetivo de verificar como o assunto tem sido estudado nas diferentes áreas de conhecimento, uma vez que o tema é interdisciplinar. As revistas científicas são consideradas o melhor suporte para a comunicação do conhecimento das diversas áreas, por meio dos artigos que são publicados após a avaliação rigorosa dos pares (Población, Witter, Ramos \& Funaro, 2011).

A produção científica é um processo contínuo, dinâmico, que envolve a descoberta e a alteração do conhecimento, a comprovação de modelos e teorias e está sempre em fase de ampliação, comprovação e reformulação. Busca, acima de tudo, partilhar seus resultados com a comunidade científica e a sociedade, como forma de democratização do conhecimento (Witter, 1996; 1997). Nas universidades brasileiras, especificamente, a produção científica concentra-se mais nos cursos de mestrado e doutorado, restringindo-se à Pós-Graduação Stricto-Sensu (Witter, 1997).

Cabe ressaltar, entretanto, que a publicação dos conhecimentos gerados pelas diferentes áreas do saber deve sempre ser feita em suportes científicos confiáveis, a fim de se construírem bases de dados sólidas e de real utilidade para toda a população (Población, Witter \& Silva, 2006; Witter, 1997). Portanto, os estudos de metaciência são importantes na medida em que a avaliação da produção de um determinado tema ou área de conhecimento permite levantar informações sobre o avanço científico, a predominância e escassez de temas investigados, o método utilizado, o delineamento da pesquisa, a análise dos dados, entre outros aspectos relevantes (Buriti, C. Witter \& G.P. Witter, 2007).

A pesquisa de Witter, Bassit, Tiscar, Ferrara e Mello (2006) é um exemplo de análise de produção científica sobre envelhecimento, na qual foram avaliadas nove revistas, cinco da área de Psicologia, duas da área de Educação e duas da área da Saúde, no período de 1999 a 2003. As revistas foram escolhidas pelo conceito A Nacional ou Internacional do sistema de classificação Qualis da Coordenação de Aperfeiçoamento de Pessoal de Nível Superior (CAPES), na época. Foram publicados 1944 artigos, sendo que apenas 52 estudos eram sobre envelhecimento (2,7\%). Desses, a maior concentração estava na área da Saúde, com 42 pesquisas (80,8\%), 
seguida pela Psicologia, com 10 trabalhos (19,2\%), e nenhum artigo na área de Educação. Houve predomínio da autoria feminina $(67,0 \%)$ e de pesquisas de levantamento $(55,8 \%)$.

O trabalho de Ferreira (2006) é outro exemplo de pesquisa de metaciência que analisou o primeiro semestre de publicação de 2003, da base de dados PsycINFO, da American Psychology Association (APA), cuja busca com a palavra-chave idoso (aged) resultou em 1281 textos. Os resultados demonstraram a predominância significante $\left(\chi_{0}^{2}=1\right.$ 123,94) de títulos entre 11 e 15 vocábulos (44,3\%); de autoria múltipla (90,1\%; $\left.\chi_{0}^{2}=823,4\right)$ e maior produtividade do sexo masculino $\left(42,5 \% ; \chi_{0}^{2}=72,78\right)$. A meta-análise feita sobre psicologia do idoso na base de dados Lilacs, de 1991 a 2003, por Almeida, Rodrigues, Buriti eWitter (2007) baseou-se nos resumos de 77 artigos capturados na base com os verbetes psicologia e idoso. Os resultados foram significantes para autoria múltipla $\left(66,0 \% ; \chi_{0}^{2}=51,01\right)$ e sexo feminino $\left(48,0 \% ; \chi_{0}^{2}=28,98\right)$.

Em virtude do exposto, foi estabelecido, como objetivo geral, analisar artigos sobre qualidade de vida em idosos, na base de dados Scientific Eletronic Library Online (SciELO) e, como objetivos específicos, analisar os artigos quanto às variáveis: (1) extensão dos títulos; (2) autoria; (3) sexo dos autores; (4) áreas de produção de conhecimento e (5) área de conhecimento dos periódicos.

\section{Método}

Foi realizado um levantamento de artigos, na base de dados SciELO, utilizando-se os descritores terceira idade, velhice, idoso, idosos e envelhecimento, todos cruzados com a palavra-chave qualidade de vida. A análise da produção científica baseou-se, assim, em 69 artigos capturados na base de dados.

Após a seleção dos artigos, todos os resumos foram lidos e analisados da seguinte forma: (1) extensão dos títulos (12 vocábulos, menos de 12 vocábulos ou mais de 12 vocábulos); (2) autoria (única, coautoria ou múltipla); (3) sexo dos autores (masculino, feminino ou indefinido); (4) áreas de produção de conhecimento (Educação Física, Nutrição, Psicologia ou Outras) e (5) área de conhecimento dos periódicos (Ciências Humanas ou Ciências da Saúde).
Posteriormente, foram elaboradas as tabelas, aplicada a estatística descritiva e não paramétrica (teste de Qui-quadrado), e os resultados encontrados foram discutidos conforme a literatura científica da área. 0 nível de significância adotado foi de 5\% $(p<0,05)$.

\section{Resultados e Discussão}

Os resultados obtidos neste trabalho estão sujeitos às limitações inerentes à técnica de levantamento da produção científica. Apesar do adequado rastreamento, a identificação de artigos depende, por exemplo, da escolha das palavras-chave determinadas pelos autores e das ferramentas de busca das bases. Portanto, algumas produções podem não ter sido identificadas, assim como alguns periódicos não estão inseridos na base pesquisada.

De acordo com Domingos (1999), Granja e Grandi (1995), a recomendação de que os títulos tenham até 12 vocábulos foi seguida por 57,9\% dos artigos, sendo que $11,6 \%$ desses continham exatamente 12 vocábulos, como, por exemplo, o trabalho de S. R. Santos I. B. C. Santos, Fernandes \& Henriques (2002), com o título "Qualidade de vida do idoso na comunidade: aplicação da Escala de Flanagan",e 46,38\% tinham menos de 12 vocábulos, tais como: Luz e Amatuzzi (2008) e Luciana Reis, Torres e Luana Reis (2008), com os títulos "Vivências de felicidade de pessoas idosas" e "Características de dor em pacientes idosos institucionalizados", respectivamente. Artigos cujos títulos ultrapassaram essa recomendação somaram 42,03\%, sendo a amplitude de 13 até 22 vocábulos, como o trabalho de Machado, Basso, Margarida e Moritz (2007), com o título"Avaliação da qualidade e satisfação de vida dos pacientes antes da internação na Unidade de Terapia Intensiva e após a alta hospitalar", e a pesquisa de Chikude, Fujiki, Hond, Ono e Milani (2007), com o título "Avaliação da qualidade de vida dos pacientes idosos com fratura do colo do fêmur tratados cirurgicamente pela artroplastia parcial do quadril" (Tabela 1).

Os resultados da pesquisa de Ferreira (2006) sobre idoso na base de dados PsycINFO encontrou dados semelhantes nos vocábulos dos títulos analisados, cuja média foi de 13,02, sendo 30,21\% até 10 vocábulos, 44,26\% entre 11 e 15 palavras (predo- 
Tabela 1

Análise de artigos sobre envelhecimento e qualidade de vida quanto à extensão dos títulos. SciELO, 2010

\begin{tabular}{lcc}
\hline Extensão do título & $\mathrm{n}$ & $\%$ \\
\hline Com 12 vocábulos & 8 & 11,6 \\
Menos de 12 vocábulos & 32 & 46,5 \\
Mais de 12 vocábulos & 29 & 42,0 \\
\hline Total & 69 & 100,0 \\
\hline
\end{tabular}

minante) e 25,53\% com 16 até mais de 26 vocábulos, considerando-se o total de 1281 publicações analisadas. Vale enfatizar que a quantidade de artigos analisados na base de dados americana é superior à da brasileira, o que requer maior atenção por parte dos autores e editores na publicação dos trabalhos, bem como dos pareceristas ao ponderarem sobre as pesquisas realizadas.

Títulos muito longos indicam a necessidade de um melhor desenvolvimento na área. Em áreas cujas pesquisas são mais escassas, os títulos, em geral, são demasiadamente longos e, por vezes, nem cabem por extenso na base, o que é incompatível com a pós-modernidade, gerando frustração e perda de possíveis leitores (C. Witter, Buriti \& G. P. Witter, 2007). O título é o primeiro contato do leitor com o artigo e deve atrair a atenção de forma a instigar a leitura do resumo, o qual precisa estar adequadamente escrito para provocar a vontade de ler o artigo na íntegra.

Os valores encontrados para o teste estatístico foram: $\chi_{0}^{2}=13,87 ; \chi_{c}^{2}=5,99 ; n g l=2$. Como o Qui-quadrado observado foi maior que o crítico, houve diferença estatisticamente significativa para a extensão dos títulos dos artigos. A maioria dos títulos tinha até 12 vocábulos (57,97\%), conforme recomendado pela APA (2001). Além da extensão, devem ser observadas outras características, tais como: clareza, criatividade e qualidade (Sabadini, Sampaio \& Koller, 2009).
Volpato (2003) destaca que o título deve atrair o leitor, dar informação clara sobre o conteúdo do trabalho, porém, sem o enganar ou aborrecer. O título não deve iludir ou criar uma expectativa no leitor que não será correspondida na leitura do texto, porque desperdiça o tempo do pesquisador com um trabalho cujo conteúdo não atende às suas necessidades de pesquisa. Isso tem também implicações éticas, uma vez que pode ser uma forma de enganar o leitor e, até mesmo, gerar custos na aquisição de matéria que não contribua efetivamente para suas necessidades.

Em relação à autoria, os artigos foram divididos em três subcategorias: única ou individual, se só um autor responsabilizou-se pelo texto; coautoria, se dois autores foram responsáveis; e múltipla, se o texto teve três ou mais autores responsáveis. Na maioria dos artigos (89,8\%) houve predomínio da autoria múltipla. Artigos em parceria ou coautoria somaram 9,8\%, como o trabalho de Sousa e Russo (2009), com o título "Audição e percepção da perda auditiva em idosos". Artigos produzidos por autoria única ou individual, como a pesquisa de Santos (2006), intitulada "Relação do sexo e da idade com nível de qualidade de vida em renais crônicos hemodialisados", somaram apenas 0,41\% do total. O teste do Qui-quadrado encontrou, para autoria: $\chi_{0}^{2}=156,44 ; \chi_{c}^{2}=3,14 ; n g l=1$, sendo excluída a categoria individual devido à baixa frequência. Portanto, foi estatisticamente significativa a diferença entre coautoria e autoria múltipla (Tabela 2).

A pesquisa de Santos et al. (2009), com o título "Situação vacinal com a qualidade de vida, a funcionalidade e a motivação para o autocuidado em idosos", é um exemplo de trabalho de múltipla autoria. Os autores investigaram a situação vacinal de idosos quanto às vacinas anti-influenza, a dupla tipo adulto, constituída

Tabela 2

Análise de artigos sobre envelhecimento e qualidade de vida quanto à autoria e sexo. SciELO, 2010

\begin{tabular}{|c|c|c|c|c|c|c|c|c|}
\hline \multirow[b]{2}{*}{ Autoria/Sexo } & \multicolumn{2}{|c|}{ Feminino } & \multicolumn{2}{|c|}{ Masculino } & \multicolumn{2}{|c|}{ Indefinido } & \multicolumn{2}{|c|}{ Total } \\
\hline & $n$ & $\%$ & $n$ & $\%$ & $n$ & $\%$ & $n$ & $\%$ \\
\hline Múltipla & 136 & 88,3 & 79 & 91,8 & 5 & 100,0 & 220 & 89,8 \\
\hline Coautoria & 18 & 11,7 & 6 & 6,9 & 0 & 0,0 & 24 & 9,8 \\
\hline Individual & 0 & 0,0 & 1 & 1,2 & 0 & 0,0 & 1 & 0,4 \\
\hline Total & 154 & 100,0 & 86 & 100,0 & 5 & 100,0 & 245 & 100,0 \\
\hline
\end{tabular}


dos toxicoides tetânicos e diftérico, e a antipneumocócica polissacarídea 23 valente, relacionando com aspectos demográficos, qualidade de vida, índice de funcionalidade e motivação para o autocuidado. Cabe ressaltar o número elevado de autores $(n=8)$ para estudar poucas variáveis.

O maior índice de autoria múltipla encontrado reflete uma tendência crescente desde os anos 1970, revelando que há um amadurecimento cientifico na área e, provavelmente, uma perspectiva multidisciplinar (Población et al., 2006; Witter, 1997). Esse resultado era esperado, uma vez que as pesquisas sobre idosos e envelhecimento defendem uma abordagem interdisciplinar sobre o fenômeno (Bassit \& Witter, 2006). As pesquisas de Ferreira (2006) e de Almeida et al. (2007) sobre o tema do idoso também encontraram predomínio significante de autoria múltipla nas bases de dados PsycINFO (90,1\%; $\left.\chi^{2}=823,4\right)$ e Lilacs $(66,0 \%$; $\left.\chi_{0}^{2}=51,01\right)$. Esse predomínio é considerado como indicativo de grupos de pesquisa que sistematicamente estudam um tema, viabilizando avanços mais consistentes e com maior rapidez.

Quanto ao sexo, houve predomínio do feminino $(62,86 \%)$, em relação ao masculino $(35,1 \%)$. Autoria indefinida (quando não foi possível identificar o sexo pelo nome, como por exemplo, Valdeci e Darci) contribuiu, apenas, com 2,04\% do total. O predomínio de participação de autoria feminina em pesquisas sobre qualidade de vida e idosos foi estatisticamente significativo $\left(\chi_{0}^{2}=135,20\right.$ e $\chi_{c}^{2}=5,99 ; n g l=2$.), possivelmente pelo fato de os trabalhos estarem concentrados em áreas do conhecimento, no Brasil, com maior concentração do sexo feminino, tanto na formação quanto na atuação, como a Psicologia (Witter, 1999).

Um exemplo de autoria exclusivamente feminina é a pesquisa "Repercussões da aposentadoria na qualidade de vida do idoso", cujo objetivo foi compreender como o idoso vivencia a aposentadoria e as suas repercussões na qualidade de vida, sendo as autoras Alvarenga, Kiyan, Bittencourt e Wanderley (2009), duas nutricionistas e duas psicólogas. O maior ou menor índice de produção científica por determinado sexo varia de acordo com a área de conhecimento, e há predomínio de determinado sexo em áreas específicas, como, por exemplo, no Brasil, maior quantidade de

398 pesquisadoras em Psicologia e Nutrição. Seria relevante conseguir tornar o fazer ciência nessas áreas e nas próprias profissões mais atrativos para o sexo masculino. As associações científicas e os conselhos profissionais poderiam promover programas neste sentido.

As pesquisas de produção científica sobre idosos e envelhecimento de Witter et al. (2006) e Almeida et al. (2007), com foco na área de Psicologia, também encontraram maior produtividade feminina, respectivamente, nas revistas científicas brasileiras (67\%; $\left.\chi_{0}^{2}=10,14\right)$ e na base de dados latino-americana Lilacs $\left(48,03 \% ; \chi_{0}^{2}=28,98\right)$. Porém, o esperado seria que essa produção fosse equitativa, havendo similaridade entre os sexos (Población et al. 2006).

Em relação à área de conhecimento (Tabela 3), a que mais produziu sobre o tema foi Psicologia (26,1\%), seguida de Medicina (21,7\%), Saúde Pública e Enfermagem (17,4\% cada) e Psiquiatria (11,6\%). Fonoaudiologia, Fisioterapia, Odontologia e Educação Física contribuíram, apenas, com 1,4\% do total, cada uma. Em nenhum dos artigos foram abordados assuntos referentes à Nutrição. $O$ teste estatístico, que revelou 0 $\chi_{0}^{2}=0,14 ; \chi_{c}^{2}=9,49 ; n g l=4$, foi aplicado para as cinco áreas com maior quantidade de publicações, excluindo-se as áreas com um único registro para efeito de cálculos. Não houve diferença estatisticamente significativa para as áreas de conhecimento dos artigos. Esse resultado revela que o tema estudado tem um caráter multidisciplinar, porque diversas áreas do conhecimento estão investigando os idosos, e muitos artigos revelam um enfoque interdisciplinar sobre o processo de envelhecimento, quando agregam mais de uma disciplina na discussão da qualidade de vida na velhice. Por exemplo, a ausência de trabalhos na área de Nutrição torna urgente a mobilização dos nutricionistas na realização de pesquisas que relacionem qualidade de vida e dieta, uma vez que ter uma boa nutrição em todas as fases do ciclo da vida é fator determinante de qualidade de vida. A alimentação é essencial para se ter uma boa saúde e um estado nutricional adequado, e é a variável externa que mais afeta a velhice (Santos \& Rezende, 2006).

As pesquisas de metaciência sobre a temática do idoso e do processo de envelhecimento têm sido objeto de estudo constante de áreas como a Psicologia e a Saúde Pública, porém, ausentes em áreas como a Educação (Witter et al., 2006). Fato semelhante foi 
Tabela 3

Análise de artigos sobre envelhecimento e qualidade de vida quanto às áreas de conhecimento. SciELO, 2010

\begin{tabular}{lcc}
\hline Área de conhecimento & $\mathrm{n}$ & $\%$ \\
\hline Psicologia & 18 & 26,1 \\
Medicina & 15 & 21,4 \\
Saúde Pública & 12 & 17,4 \\
Enfermagem & 2 & 17,6 \\
Psiquiatria & 8 & 11,6 \\
Fonoaudiologia & 1 & 1,4 \\
Fisioterapia & 1 & 1,4 \\
Odontologia & 1 & 1,4 \\
Educação Física & 1 & 1,4 \\
\hline Total & 69 & 100,0 \\
\hline
\end{tabular}

observado no presente trabalho sobre qualidade de vida, idoso e envelhecimento, no qual as áreas de Psicologia, Medicina, Saúde Pública, Enfermagem e Psiquiatria foram as que mais produziram sobre o assunto, enquanto Fonoaudiologia, Fisioterapia, Odontologia e Educação Física contribuíram com um único artigo cada uma, e nenhum sobre qualidade de vida e Nutrição foi encontrado.

Como exemplos, podem-se citar a pesquisa de Carneiro, Falcone, Clark, Z. Del Prette e A. Del Prette, (2007), com o título "Qualidade de vida, apoio social e depressão em idosos: relação com habilidades sociais", da "Revista Psicologia: Reflexão e Crítica"; a pesquisa de Brandão, Nascimento e Viana (2009), com o título "Capacidade funcional e qualidade de vida em pacientes idosos com ou sem disfagia após acidente vascular encefálico isquêmico", da "Revista da Associação Médica Brasileira"; a pesquisa de Martins, Barreto e Pordeus (2009), intitulada "Auto-avaliação de saúde bucal em idosos: análise com base em modelo multidimensional, do periódico Cadernos de Saúde Pública"; o trabalho de Kusumoto, Marques, Haas e Rodrigues (2008), com o título "Adultos e idosos em hemodiálise: avaliação da qualidade de vida relacionada à saúde", da "Revista Acta Paulista de Enfermagem"; e a pesquisa de Paula, Roque e Araujo (2008), com o título "Qualidade de vida em cuidadores de idosos portadores de demência de Alzheimer", do "Jornal Brasileiro de Psiquiatria". Os artigos foram publicados em diversas revistas científicas dessas áreas de conhecimento, principalmente Medicina, fato que pode ser reflexo da carência de periódicos indexados na SciELO com conteúdo voltado, exclusivamente, para esse segmento da população.

A população idosa é a que mais cresce no Brasil e, consequentemente, tem merecido a atenção de diversas áreas do conhecimento. Portanto, a produção científica sobre essa temática precisa ser maior e mais bem divulgada, tanto no meio acadêmico e científico como na sociedade em geral e nas revistas de divulgação. É importante destacar a necessidade de criação e indexação de novas revistas científicas especializadas em idosos e no processo de envelhecimento, porque atualmente não existe nenhuma na base de dados da SciELO (2012). Na WebQualis da Coordenação de Aperfeiçoamento de Pessoal de Nível Superior, existem seis periódicos brasileiros e um espanhol cujos títulos contêm a palavra gerontologia, e duas revistas com a palavra envelhecimento, sendo que o melhor no estrato da classificação dos periódicos é B1 Capes (2012). Isso revela que os pesquisadores e a área de estudos do envelhecimento precisam continuar amadurecendo, inclusive para a criação de revistas científicas que possam melhorar e concentrar a divulgação das suas pesquisas com foco no idoso e no envelhecimento, sem que se perca espaço nas demais.

O idoso é o público mais susceptível a doenças e agravos não transmissíveis, como, por exemplo, doenças cardiovasculares, diabetes e hipertensão arterial, que comprometem sua saúde e seu bem-estar, o que justificaria uma maior produção de conhecimentos em áreas como Nutrição e Educação Física, as quais poderiam colaborar com mais pesquisas sobre prevenção dessas doenças e manutenção da qualidade de vida na velhice. Há uma tendência de que as áreas de conhecimento se integrem no estudo do idoso e do envelhecimento humano e que a produção científica tenha um enfoque cada vez mais interdisciplinar, como, por exemplo, no projeto sobre Fragilidade em Idosos Brasileiros (FIBRA), que reuniu diversas áreas (Neri, 2007b; Neri \& Guariento, 2011).

Quanto à área de conhecimento dos periódicos (Tabela 4), os artigos foram divididos em duas subcategorias: Ciências Humanas e Ciências da Saúde, sendo que a primeira contribuiu com 14,5\% das produções, e a segunda, com 85,5\%. Houve diferença estatisticamente significativa entre a área de Ciências da Saúde e de Ciências Humanas $\left(\chi_{0}^{2}=33,79 ; \chi_{c}^{2}=3,14\right.$; ngl=1), pro- 


\section{Tabela 4}

Análise de artigos sobre envelhecimento e qualidade de vida quanto à área de conhecimento dos periódicos. SciELO, 2010

\begin{tabular}{|c|c|c|}
\hline Área de conhecimento & $n$ & $\%$ \\
\hline \multicolumn{3}{|l|}{ Ciências Humanas } \\
\hline Psicologia em Estudo & 3 & 4,3 \\
\hline Interface: Comunicação, Saúde e Educação & 2 & 2,9 \\
\hline Estudos de Psicologia (Campinas) & 2 & 2,9 \\
\hline Psicologia: Reflexão e Critica & 1 & 1,4 \\
\hline Psicologia Clínica & 1 & 1,4 \\
\hline Psicologia USP & 1 & 1,4 \\
\hline Subtotal & 10 & 14,5 \\
\hline \multicolumn{3}{|l|}{ Ciências da Saúde } \\
\hline Revista de Saúde Pública & 12 & 17,8 \\
\hline Revista Brasileira de Psiquiatria & 5 & 7,2 \\
\hline Revista Latino-Americana de Enfermagem & 4 & 5,8 \\
\hline Cadernos de Saúde Pública & 4 & 5,8 \\
\hline Acta Paulista de Enfermagem & 4 & 5,8 \\
\hline Revista da Sociedade Brasileira de Fonoaudiologia & 3 & 4,3 \\
\hline Revista da Associação Médica Brasileira & 3 & 4,3 \\
\hline Revista Brasileira de Enfermagem & 3 & 4,3 \\
\hline Revista do Hospital das Clinicas & 2 & 2,9 \\
\hline Revista da Escola de Enfermagem da USP & 2 & 2,9 \\
\hline Revista Brasileira de Otorrinolaringologia & 2 & 2,9 \\
\hline Revista Brasileira de Epidemiologia & 2 & 2,9 \\
\hline Outros & 13 & 18,8 \\
\hline Subtotal & 59 & 85,51 \\
\hline Total & 69 & 100,0 \\
\hline
\end{tabular}

vavelmente devido ao fato de as Ciências Biomédicas investigarem o envelhecimento há mais tempo e pela colaboração científica mais presente na discussão da conceituação de qualidade de vida (WHO, 1998). Além disso, a Psicologia é compreendida como uma área de conhecimento das Ciências Humanas e da Saúde, uma vez que a atuação do profissional ocorre na área da Saúde, em particular, da saúde mental (Witter, 1999) ou psicossocial.

O periódico com maior representatividade foi a "Revista de Saúde Pública"(17,4\%), tal como se verificou na pesquisa de Alexandre, Cordeiro e Ramos (2009), cujo objetivo foi analisar se a qualidade de vida de idosos ativos e saudáveis pode ser influenciada por estado funcional, características sociodemográficas e parâmetros psicológicos. O segundo periódico mais representativo foi a "Revista Brasileira de Psiquiatria" (7,2\%). Como exemplo, cita-se a pesquisa de Antunes, Stella, Santos, Bueno \& Mello (2005), que objetivou examinar o efeito de um programa de exercício aeróbio na intensidade do limiar ventilatório 1 nos escores indicativos de depressão e ansiedade e na qualidade de vida dos idosos saudáveis.

A "Revista Latino-Americana de Enfermagem", os "Cadernos de Saúde Pública" e a "Acta Paulista de Enfermagem" somaram 5,8\% do total das publicações cada um, sendo todos da área de Ciências da Saúde. Na categoria "Outros" ficaram agrupados os periódicos da área de Ciências da Saúde que apareceram com baixa frequência: um único registro. Os três periódicos de "Psicologia: Reflexão e Crítica, Psicologia Clínica" e "Psicologia USP", embora também tenham aparecido uma única vez, foram citados porque, dentre os periódicos da área de Ciências Humanas, poucas revistas foram encontradas, não justificando a designação "Outros".

A meta-análise de Witter et al. (2006) analisou, na área da Saúde, a "Revista de Saúde Pública" e os "Cadernos de Saúde Pública" e, na área da Psicologia, os periódicos "Psicologia: Reflexão e Crítica","Estudos de Psicologia", "Psicologia: Ciência e Profissão e Psicologia USP", de 1999 até 2003, tendo obtido resultados semelhantes. Dos 52 artigos sobre idoso e envelhecimento, 42 foram publicados nas revistas da área da Saúde e apenas dez na área de Psicologia. Portanto, houve uma diferença significativa na produtividade, com predomínio da área da Saúde.

Esta pesquisa limitou-se ao estudo dos aspectos mais formais da comunicação científica, porém, que permitem analisar parte da evolução do conhecimento na área. A realização de pesquisas sobre produção científica, em especial, de artigos e de revistas científicas, consolidaram a sua importância na avaliação do conhecimento e da comunicação científica (Población et al., 2011), tendo ampliado as variáveis de estudo e aprimorado a metodologia de trabalho até as pesquisas de revisão sistemática e meta-análise com ensaios clínicos randomizados (Borenstein, Hedges, Higgins \& Rothstein, 2009). Portanto, a temática do idoso e qualidade de vida merece a análise de outras variáveis dos artigos publicados, tais como: os aspectos descritivos do método (participantes, materiais, procedimentos); tipo de análise dos resultados (qualitativos e/ou quantitativos; descritivos e/ou inferenciais); tipo de delineamento das pesquisas (levantamento, correlacional, quase experimental e experimental); principais resul- 
tados e conclusões; entre outras possibilidades relevantes de investigação. Também, seria relevante pesquisar as características específicas do discurso científico nas várias áreas e bases de dados.

\section{Considerações Finais}

É importante considerar que se observa nos últimos anos um maior número de publicações relacionadas ao envelhecimento, e o aumento da produção de artigos científicos pode representar um maior número de pesquisadores e, ainda, incentivo do governo em políticas de apoio da pesquisa científica e formação de recursos humanos para a pesquisa no País.

A partir da análise dos resultados encontrados, pôde-se observar predomínio de títulos com menos de 12 vocábulos, de autoria múltipla e feminina. As áreas que mais produziram sobre o tema foram Psicologia e Medicina, e o periódico com maior representatividade foi a "Revista de Saúde Pública", integrante da área de Ciências da Saúde. É necessário que a área de Ciências Humanas mobilize-se para aumentar a produção de pesquisas direcionadas ao envelhecimento, em particular, sobre qualidade de vida do idoso, uma vez que se trata de um fenômeno crescente em todo o mundo. O tema merece, de fato, mais atenção dos que geram conhecimento sobre o ser humano e suas relações pessoais, sociais e culturais.

Observou-se uma grande variabilidade de questões e temas estudados nas pesquisas sobre qualidade de vida e envelhecimento, com grande dispersão dos resultados, dificultando uma análise mais profunda dos artigos levantados. Entretanto, sugere-se que sejam estudadas as variáveis metodológicas, de delineamento, de análise de dados, dos resultados e da temática, de modo a permitir um aprofundamento e cruzamento das variáveis comuns e divergentes das pesquisas, na tentativa de definir diretrizes para o fomento e a produção de conhecimento na área do envelhecimento.

Certamente, há necessidade de maior produção de trabalhos vinculados a áreas de pesquisa com linhas bem definidas para um desenvolvimento mais sistemático e produtivo. Nessas circunstâncias, políticas específicas de grupo, institucionais e mesmo mais abrangentes precisam ser estabelecidas, por exemplo, pelos conselhos profissionais federais e regionais das diversas áreas de conhecimento como Psicologia - Conselho Federal de Psicologia (CFP) e Conselho Regional de Psicologia (CRP); Nutrição - Conselho Federal de Nutricionistas (CFN) e Conselho Regional de Nutricionistas (CRN); Fisioterapia - Conselho Federal de Fisioterapia e Terapia Ocupacional (COFFITO) e Conselho Regional de Fisioterapia e Terapia Ocupacional (CREFITO); etc., pelas instituições de ensino superior e de pesquisa que destinam recursos financeiros e humanos para o estudo do processo de envelhecimento.

Vale ressaltar, ainda, a necessidade de realização de estudos futuros com temas importantes já consagrados na literatura científica, como, por exemplo, a depressão e as doenças crônicas (cardiovasculares e diabetes) que surgem no processo de envelhecimento, porém com o levantamento de estudos de casos clínicos randomizados que permitam um tratamento estatístico mais aprofundado e próprio das pesquisas de meta-análise. Nestas pesquisas, são investigados os seguintes aspectos: as variáveis independentes e dependentes; o método com o detalhamento dos participantes, materiais e procedimentos; os resultados e conclusões. As pesquisas de meta-análise permitem determinar os estudos mais relevantes e avaliar os tratamentos e medicamentos mais eficazes. Vale lembrar que ensaios clínicos precisam ser registrados, sendo que anteriormente isto só podia ocorrer em bases estrangeiras e que agora o país já conta com o Registro Brasileiro de Ensaios Clínicos (REBEC), o que pode proporcionar maior compreensão dos problemas assegurando qualidade científica.

É importante esclarecer que temas mais subjetivos, como sobre qualidade de vida, produzem um conhecimento por vezes mais limitado, como o número reduzido de participantes e com delineamento mais qualitativo ou mesmo quantitativo cuja metodologia não permite a realização de um trabalho de meta-análise porque as pesquisas produzidas não são estudos de casos clínicos randomizados. Embora determinadas temáticas não permitam a realização de análises mais profundas, inclusive estatísticas, as revisões de literatura e sistemáticas são fundamentais para a avaliação do conhecimento de um determinado tema ou área na tentativa de promover não apenas o avanço 
científico, mas também a prática profissional baseada em evidências científicas.

Também não se pode esquecer em relação ao tema analisado, até para se ter um bom parâmetro de comparação, o estado de idosos com boa qualidade de vida, com êxito no enfrentamento das perdas e bom uso do que há de positivo no envelhecer. É necessária a varredura e avaliação do conhecimento produzido em uma determinada área, como a Psicologia, nas diversas bases de dados nacionais e estrangeiras para a realização de uma revisão sistemática sobre a qualidade de vida de idosos no processo de envelhecimento. Essa é uma atividade científica cada vez mais necessária para o planejamento e a definição de diretrizes de políticas científicas, de fomento para as pesquisas e os grupos de pesquisadores, assim como para a avaliação da qualidade da produção científica, consolidação e avanços, sobre um determinado tema, determinada área de conhecimento e, até mesmo, de um grupo de pesquisa ou de um pesquisador.

\section{Referências}

Alexandre, T. S., Cordeiro, R. C., \& Ramos, L. R. (2009). Fatores associados à qualidade de vida em idosos ativos. Revista de Saúde Pública, 43(4), 613-621.

Almeida, L. A., Rodrigues, L. O., Buriti, M. A., \& Witter, G. P. (2007). Meta-análise da produção científica sobre psicologia do idoso no Lilacs (1991-2003). In M. A. Buriti, C. Witter \& G. P. Witter (Orgs.), Produção científica e psicologia educacional (pp.57-78). Guararema: Anadarco.

Alvarenga, L. N., Kiyan, L., Bittencourt, B., \& Wanderley, K. S. (2009). Repercussões da aposentadoria na qualidade de vida do idoso. Revista da Escola Paulista de Enfermagem da USP, 43(4), 796-802.

American Psychological Association. (2001). Publication manual of American Psychological Association (5 $5^{\text {th }}$ ed.). Washington, DC: Author.

Antunes, H. K. M., Stella, S. G., Santos, R. F., Bueno, O. F. A., \& Mello, M. T. (2005). Depression, anxiety and quality of life scores in seniors after endurance exercises program. Revista Brasileira de Psiquiatria, 27(4), 266-271.

Baltes, P. B., \& Smith, J. (2006). Novas fronteiras para o futuro do envelhecimento: a velhice bem-sucedida do idoso jovem aos dilemas da quarta idade. A Terceira Idade, 17(36), 7-31.

Bassit, A. Z., \& Witter, C. (2006). Envelhecimento: objeto de estudo e campo de intervenção. In G. P. Witter (Org.), Envelhecimento: referenciais teóricos e pesquisas. Campinas: Alínea.
Borenstein, M., Hedges, L. V., Higgins, J. P. T., \& Rothstein, H. R. (2009). Introduction to meta-analysis. West Sussex, UK: Wiley.

Borglin, G., Edberg, A. K., \& Hallberg, I. R. (2005). The experience of quality of life among older people. Journal of Aging Studies, 19(2), 201-220.

Brandão, D. M. S., Nascimento, J. L. S., \& Vianna, L. G. (2009). Capacidade funcional e qualidade de vida em pacientes idosos com ou sem disfagia após acidente vascular encefálico isquêmico. Revista da Associação Médica Brasileira, 55(6), 738-743.

Buriti, M. A., Witter, C., \&Witter, G. P. (Orgs.). (2007). Produção científica e Psicologia Educacional. Guararema: Anadarco.

Carneiro, R. S., Falcone, E., Clark, C., Del Prette, Z., \& Del Prette, A. (2007). Qualidade de vida, apoio social e depressão em idosos: relação com habilidades sociais. Psicologia: Reflexão e Crítica, 20(2), 229-237.

Cervato, A. M., Derntl, A. M., Latorre, M. R. O., \& Marucci, M. F. N. (2005). Educação nutricional para adultos e idosos: uma experiência positiva em Universidade Aberta para Terceira Idade. Revista de Nutrição, 18(1), 41-52. doi: 10.15 90/S1415-52732005000100004.

Chikude, T., Fujiki, E. N., Hond, E. K., Ono, N. K., \& Milani, C. (2007). Avaliação da qualidade de vida dos pacientes idosos com fratura do colo do fêmur tratados cirurgicamente pela artroplastia parcial do quadril. Acta Ortopédica Brasileira, 15(4), 197-199.

Coordenação de Aperfeiçoamento de Pessoal de Nível Superior. (2012). Webqualis: consulta de periódicos. Recuperado em abril 30, 2012, disponível em <http://qualis. capes.gov.br/webqualis/ConsultaPeriodicos.faces>.

Debert, G. G. (1999). A reinvenção da velhice: socialização e processos de reprivatização do envelhecimento. São Paulo: EDUSP.

Domingos, N. A. M. (1999). Análise da estrutura dos resumos de dissertações e teses em psicologia. In G. P. Witter (Org.), Produção científica em psicologia e educação (pp.47-78). Campinas: Alínea.

Granja, E. C., \& Grandi, M. E. (1995). Resumos: teoria e prática. São Paulo: Serviço de Biblioteca e Documentação do Instituto de Psicologia da USP.

Ferreira, A. A. (2006). Produção científica sobre idoso na PsycINFO (2003). In G. P. Witter (Org.), Envelhecimento: referenciais teóricos e pesquisas (pp.177-210). Campinas, SP: Alínea.

Kalache, A., Veras, R. P., \& Ramos, L. B. (1987). O envelhecimento da população mundial: um desafio novo. Revista de Saúde Pública, 21(3), 200-210.

Kusumoto, L., Marques, S., Haas, V. J., \& Rodrigues, R. A. P. (2008). Adultos e idosos em hemodiálise: avaliação da qualidade de vida relacionada à saúde. Acta Paulista de Enfermagem, 21(Esp.), 152-159.

Lima, A. M. M. (2003). Saúde e envelhecimento: o autocuidado como questão (Tese de doutorado não-publicada). Programa de Pós-Graduação em Ciências, Universidade de São Paulo.

Lima, A. M. M., Silva, H. S., \& Galhardoni, R. (2008). Envelhecimento bem-sucedido: trajetórias de um constructo e novas fronteiras. Interface, 12(27), 795-807. 
Luz, M. M. C., \& Amatuzzi, M. M. (2008). Vivências de felicidade de pessoas idosas. Estudos de Psicologia (Campinas), 25(2), 303-307. doi: 10.1590/S0103-166X2008000200014.

Machado, F. O., Basso, G., Margarida, C. S., \& Moritz, R. C. (2007). Avaliação da qualidade e satisfação de vida dos pacientes antes da internação na Unidade de Terapia Intensiva e após a alta hospitalar. Revista Brasileira de Terapia Intensiva, 19(1), 60-66.

Martins, A. M. E. B. L., Barreto, S. M., \& Pordeus, I. A. (2009). Auto-avaliação de saúde bucal em idosos: análise com base em modelo multidimensional. Cadernos de Saúde Pública, 25(2), 421-435.

Neri, A. L. (Org.). (2003). Qualidade de vida na idade madura (5 ed.). Campinas: Papirus.

Neri, A. L. (Org.). (2006). Palavras-chave em gerontologia. Campinas: Átomo-Alínea.

Neri, A. L. (Org.). (2007a). Idosos no Brasil: vivências, desafios e expectativas na terceira idade. São Paulo: Fundação Perseu Abramo.

Neri, A. L. (Org.). (2007b). Qualidade devida na velhice:enfoque multidisciplinar. Campinas: Alínea.

Neri, A. L. (2007c). Qualidade de vida na velhice e subjetividade. In A. L. Neri (Org), Qualidade de vida na velhice: enfoque multidisciplinar. Campinas: Alínea.

Neri, A. L., Yassuda, M. S., \& Cachioni, M. (2004). Velhice bem-sucedida: aspectos afetivos e cognitivos. Campinas: Papirus.

Neri A. L., \& Guariento, M. E. (Orgs.). (2011). Fragilidade, saúde e bem-estar em idosos: dados do estudo FIBRA Campinas. Campinas: Alínea.

Organização Mundial da Saúde. (2005). Envelhecimento ativo: uma política de saúde. Brasília: Organização Pan-Americana de Saúde.

Paula, J. A., Roque, F. P., \& Araújo, F. S. (2008). Qualidade de vida em cuidadores de idosos portadores de demência de Alzheimer. Jornal Brasileiro de Psiquiatria, 57(4), 283-287.

Población, D. A., Witter, G. P., \& Silva, J. F. M. (Orgs.). (2006). Comunicação e produção científica: contexto, indicadores, avaliação. São Paulo: Aguillera.

Población, D. A., Witter, G. P., Ramos, L. M. S. V. C., \& Funaro, V. M. B. O. (Orgs.). (2011). Revistas científicas: dos processos tradicionais às perspectivas alternativas de comunicação. São Paulo: Ateliê.

Reis, L. A., Torres, G. V., \& Reis, L. A. (2008). Pain Characterization in institutionalized elderly patients. Arquivos deNeuro-Psiquiatria, 66(2b), 331-335.

Sabadini, A. A. Z. P., Sampaio, M. I. C., \& Koller, S. H. (2009). Preparando um artigo científico. In A. A. Z. P. Sabadini, M. I. C. Sampaio \& S. H. Koller (Orgs.), Publicar em psicologia: um enfoque para a revista científica. São Paulo: Associação Brasileira de Editores Científicos de Psicologia.
Santos, B. R. L., Creutzberg, M., Cardoso, R. F. M. L., Lima, S. F., Gustavo, A. S., \& Viegas, K., et al. (2009). A situação vacinal e associação com qualidade de vida, a funcionalidade e a motivação para ao autocuidado em idosos. Revista Brasileira de Epidemiologia, 12(4), 533-540.

Santos, P. R. (2006). Relação do sexo e da idade com nível de qualidade de vida em renais crônicos hemodialisados. Revista da Associação Médica Brasileira, 52(5), 356-359.

Santos, S. R., Santos, I. B. C., Fernandes, M. G. M., \& Henriques, M. E. R. M. (2002). Qualidade de vida do idoso na comunidade: aplicação da Escala de Flanagem. Revista Latino-Americana de Enfermagem, 10(6), 757-764.

Santos, V. H., \& Rezende, C. H. A. (2006). Nutrição e envelhecimento. In E. V. Freitas, et al. (Orgs.), Tratado de geriatria e gerontologia. Rio de Janeiro: Guanabara Koogan.

Scientific Electronic Library Online. (2012). Índice de assunto. São Paulo: Autor. Recuperado em abril 30, 2006, disponível em <http://www.scielo.br/scielo.php?script=sci_subject \&lng $=p t \& n r m=i s o \# s u b j 2>$.

Sousa, M. G. C., \& Russo, I. C. P. (2009). Audição e percepção da perda auditiva em idosos. Revista da Sociedade Brasileira de Fonoaudiologia, 14(2), 241-246.

Vecchia, R. D., Ruiz, T., Bocchi, S. C. M., \& Corrente, J. E. (2005). Qualidade de vida na terceira idade: um conceito subjetivo. Revista Brasileira de Epidemiologia, 8(3), 246-52.

Volpato, G. L. (2003). Publicação científica. São Paulo: Tipomic.

Witter, C. (1996). Psicologia escolar: produção científica, formação e atuação (1990-1994) (Tese de doutorado não- publicada). Programa de Pós-Graduação em Psicologia, Universidade de São Paulo.

Witter, G. P. (Org.). (1997). Produção científica. Campinas: Átomo.

Witter, C. (Org.). (1999). Ensino de psicologia. Campinas: Alínea.

Witter, C., Bassit, A. Z., Tiscar, D., Ferrara, J. N., \& Melo, M. V. N. R. (2006). Produção científica na delimitação de um campo de estudo: o envelhecimento. In G. P. Witter (Org.), Envelhecimento: referenciais teóricose pesquisas (pp.211-235). Campinas: Alínea.

Witter, C., Buriti, M. A., \& Witter, G. P. (Orgs.). (2007). Problemas psicossociais: análise de produção. Guararema: Anadarco.

Witter, C., \& Buriti, M. A. (2011). (Orgs.), Envelhecimento e contingências de vida. Campinas: Alínea.

World Health Organization. (1998). The World Health Organization Quality of Life assessment (WHOQOL): Development and general psychometric properties. Social, Science and Medicine, 46(12), 1569-1585.

Recebido em: 22/3/2011

Versão final em: 18/5/2012

Aprovado em: 5/6/2012 